\title{
Defect spectroscopy of single ZnO microwires
}

\author{
M. Villafuerte, ${ }^{1}$ J. M. Ferreyra, ${ }^{2}$ C. Zapata, ${ }^{2}$ J. Barzola-Quiquia, ${ }^{3}$ F. likawa, ${ }^{4}$ P. Esquinazi, ${ }^{3}$ \\ S. P. Heluani, ${ }^{2}$ M. M. de Lima, Jr., ${ }^{5}$ and A. Cantarero ${ }^{5}$ \\ ${ }^{1}$ Laboratorio de Física del Sólido, Dpto. de Física, FCEyT, Universidad Nacional de Tucumán and CONICET, \\ 4000 Tucumán, Argentina \\ ${ }^{2}$ Laboratorio de Física del Sólido, Dpto. de Física, FCEyT, Universidad Nacional de Tucumán, \\ 4000 Tucumán, Argentina \\ ${ }^{3}$ Division of Superconductivity and Magnetism, University of Leipzig, D-04103 Leipzig, Germany \\ ${ }^{4}$ Instituto de Física "Gleb Wataghin," Unicamp, 13083-859 Campinas-SP, Brazil \\ ${ }^{5}$ Materials Science Institute, University of Valencia, P.O. Box 22085, E-46071 Valencia, Spain
}

(Received 12 February 2014; accepted 14 March 2014; published online 1 April 2014)

The point defects of single $\mathrm{ZnO}$ microwires grown by carbothermal reduction were studied by microphotoluminescence, photoresistance excitation spectra, and resistance as a function of the temperature. We found the deep level defect density profile along the microwire showing that the concentration of defects decreases from the base to the tip of the microwires and this effect correlates with a band gap narrowing. The results show a characteristic deep defect levels inside the gap at $0.88 \mathrm{eV}$ from the top of the VB. The resistance as a function of the temperature shows defect levels next to the bottom of the $\mathrm{CB}$ at $110 \mathrm{meV}$ and a mean defect concentration of $4 \times 10^{18} \mathrm{~cm}^{-3}$. This combination of techniques allows us to study the band gap values and defects states inside the gap in single $\mathrm{ZnO}$ microwires and opens the possibility to be used as a defect spectroscopy method. @ 2014 AIP Publishing LLC. [http://dx.doi.org/10.1063/1.4869555]

\section{INTRODUCTION}

Zinc oxide $(\mathrm{ZnO})$ is a wide band gap semiconductor with potential applications in optoelectronics, transparent electronics, spintronic, as well as in biomedicine due to its biocompatibility character. It is known that intrinsic and extrinsic defects play a very important role in its properties and that there are many difficulties to control the dope type and characterize the defects, which they have still been the obstacles and challenges.

Normally, the n-type behavior in undoped $\mathrm{ZnO}$ is explained by the presence of native donor centers, usually identified as zinc interstitial, $\mathrm{Zn}_{i}$ or oxygen vacancies, $\mathrm{V}_{O}{ }^{1-3}$ Conversely, the formation energy of the $\mathrm{Zn}_{i}$ is very high in n-type $\mathrm{ZnO}$, and it was argued that $\mathrm{V}_{O}$ is a deep rather a shallow donor. ${ }^{4}$ Furthermore, it has been conjectured that interstitial hydrogen in the donor state, $\mathrm{H}+$, could be responsible for the electron concentration frequently observed in this material. ${ }^{5}$

The study of the spatial distribution of the defects on the surfaces, interfaces, and bulk of nano and micro-structures is an important issue for tailoring the optoelectronic properties of devices. ${ }^{6}$ Moreover, it is well known from mechanical properties of whiskers that the number of defects decreases with size in these structures. ${ }^{7}$ The latter correlation is explained considering that as diameter decreases, the surface approaches the bulk where defects and impurities can segregate during the growth of the nanostructures, leaving the bulk with a relatively small concentration of defects. ${ }^{8}$

One of most used techniques to investigate the defects in $\mathrm{ZnO}$ is the photoluminescence (PL). The typical PL spectra present usually two emission bands: One at the UV region located near the absorption edge of the crystal and other broad band at the visible region. The latter is normally in the green spectra range and is referred as the green luminescence (GL) band. Its origin is attributed to diverse native point defects such as $\mathrm{V}_{O},{ }^{9,10}$ zinc vacancy $\left(\mathrm{V}_{Z n}\right),{ }^{11,12}$ oxygen antisite $\left(\mathrm{O}_{Z n}\right),{ }^{13}$ and $\mathrm{Zn}_{i}{ }^{14}$ or their complexes. ${ }^{15}$ Extrinsic point defects were also proposed such as $\mathrm{Cu}$ impurities. ${ }^{16,17}$ The relative intensity of the two emission bands $\left(\mathrm{I}_{G L} / \mathrm{I}_{U V}\right.$, also gives worth information. At the nano-scale where the surface to volume ratio is large, the ratio of volumes takes place the deep level emission (GL) to the UV emission $\left(\mathrm{V}_{G L} / \mathrm{V}_{U V}\right.$ dominates the $\mathrm{I}_{G L} / \mathrm{I}_{U V}$ ratio, since the deep level emitting defects are localized on the surface, and the UV emission comes from the bulk. ${ }^{8}$ Nevertheless, when the density of deep level defects varies along the sample or the fluence or energy of the laser (i.e., the density of excitons) used to excite the sample, varies, it is necessary to take into account the quantum efficiencies to the production of photons of UV and GL, because its relative intensity depends on the relative numeric density of excitons and green-emitting defects. ${ }^{18}$ Since the minimum spatial resolution in PL is usually limited to about $1 \mu \mathrm{m}$, the most of the published nanowire PL spectra were the integrated response of many nanowires. The study of single nanowires or microwires (MWs) opens the possibility to investigate in detail spatial distribution (in micrometer scale) of the defect concentration, band gap narrowing/widening or other properties, using optical techniques combined with other experiments.

In this work, we report the study of defects in single $\mathrm{ZnO}$ microwires combining different experiments. Using microphotoluminescence ( $\mu \mathrm{PL})$, we scan the variation of the defect concentration and the band gap value along the microwire. Measuring the dependence of the photoconductivity on the wavelength of the light and the resistance as a function of the temperature, we could study the relation of these dependences with the point defects. Correlating the 


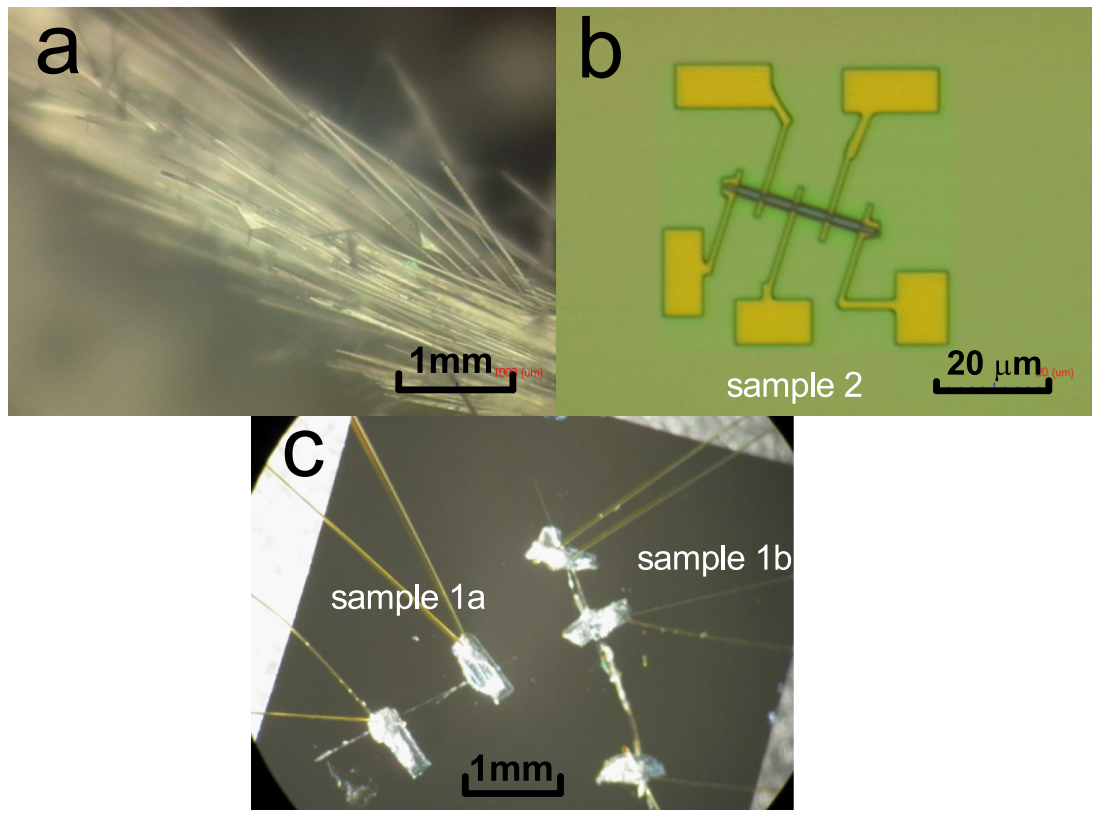

FIG. 1. (a) Photography of microwires obtained by carbothermal evaporation. (b) Sample 2 contacted by nanolithography technique. (c) Photography of samples 1(a) and 1(b): Microwires contacted by cold soldering technique using In.

information of the $\mu \mathrm{PL}$ with the photoconductivity (PC), we are able to distinguish deep states (acceptors and traps), since electrons, retained there, can be promoted to the Conduction Band (CB) by photoexcitation left aside the possibility of thermal activation. The procedure has been successfully used as a defect spectroscopy.

\section{EXPERIMENTAL DETAILS}

MWs of $\mathrm{ZnO}$ were prepared by carbothermal process consistent in the thermal decomposition of the high melting point $\mathrm{ZnO}\left(1975^{\circ} \mathrm{C}\right)$ into low melting point $\mathrm{Zn}$ or $\mathrm{Zn}$ suboxides $\left(\approx 419^{\circ} \mathrm{C}\right)$ described by the following reactions:

$$
\begin{gathered}
2 \mathrm{ZnO}(s)+C(s) \rightarrow 2 \mathrm{Zn}(g)+\mathrm{CO}_{2}(g), \\
2 \mathrm{Zn}(g)+\mathrm{O}_{2}(g) \rightarrow 2 \mathrm{ZnO}(s) .
\end{gathered}
$$

For carbothermal evaporation, ${ }^{19}$ a pressed $\mathrm{ZnO} /$ graphite target (mass ratio of 1:1) was placed on a ceramic holder inside a quartz tube. When the temperature of a programmable tubular furnace had reached $1150{ }^{\circ} \mathrm{C}$, the quartz tube was inserted into it. Both outlets of the quartz tube were kept open to the ambient air and no carrier gas was applied. The reaction time was $1 \mathrm{~h}$. The wires grow directly on the targets with diameter from 0.2 to $13 \mu \mathrm{m}$ and lengths of some millimeters (see Fig. 1(a)). A group of microwires were selected to perform $\mu \mathrm{PL}$ and others to be contacted for transport measurement with two different procedures: Nanolithography and by cold soldering (manual mechanical contacts). The Fig. 1(b) shows a microwire contacted using nanolithography. For cold soldering, $\mathrm{ZnO}$ microwires were separated and placed on a $\mathrm{Si} / \mathrm{Si}_{3} \mathrm{~N}_{4}$ substrate. Indium was employed in order to solder $\mathrm{Au}$ wires to the microwires (see Fig. 1(c)).

\section{RESULTS AND DISCUSSION}

$\mu \mathrm{PL}$ was measured in several $\mathrm{ZnO}$ microwires at $300 \mathrm{~K}$. The microwires were excited by the $325 \mathrm{~nm}$ line of a $\mathrm{HeCd}$ laser focused to a minimum spot size of $1.5 \mu \mathrm{m}$. Figure 2 shows the $\mu \mathrm{PL}$ spectra measured at different positions, marked from 1 to 4 , in a single microwire. The diameter of the microwire decreases from the base (position 1) to the tip (see inset of Fig. 2). We observe two main peaks, as mentioned above, in the UV and GL bands. The deep level defects related band (GL band) is broad from around 2.10 to $2.58 \mathrm{eV}$ and peaking at $2.37 \mathrm{eV}$ (see Fig. 2). Its intensity relative to the UV band $\left(\mathrm{I}_{G L} / \mathrm{I}_{U V}\right.$, changes along the wire (see Fig. 3), but its position remains constant within the large broadening of this band.

As proposed by Shalish et al. ${ }^{8}$ the $\mathrm{I}_{G L} / \mathrm{I}_{U V}$ ratio can be evaluated as

$$
\frac{I_{G L}}{I_{U V}}=\frac{H\left(\lambda_{G L}\right) \eta_{G L} V_{G L}}{H\left(\lambda_{U V}\right) \eta_{U V} V_{U V}},
$$

where $\eta_{G L}$ and $\eta_{U V}$ are the processes quantum efficiencies of GL and UV emissions, respectively. $\mathrm{H}(\lambda)$ is a constant that accounts for the transmission of the optical system at the corresponding wavelength $\lambda$, for our system $H\left(\lambda_{G L}\right) / H\left(\lambda_{U V}\right)$ $\approx 4 / 3$. We assume that $V_{G L} / V_{U V} \approx 0.3$ is a constant along

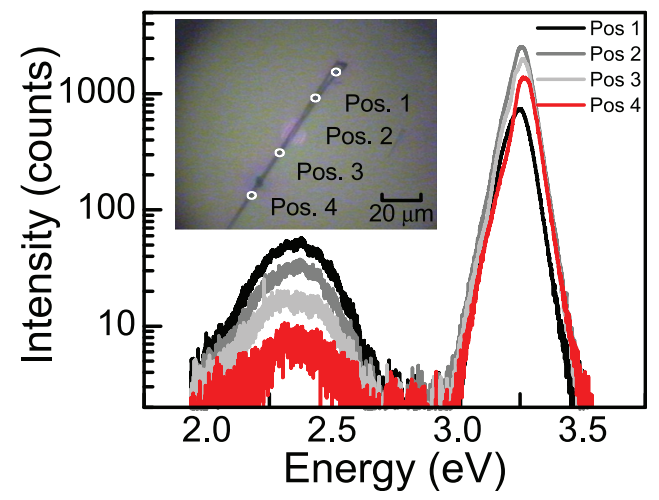

FIG. 2. Microphotoluminescence spectra at different positions in a single microwire. The inset shows the optical image of the microwire and the points indicate the position where the PL spectra were measured. One more spot (pos. 5) was taken in the thinner part of the microwire (not shown in the picture). 


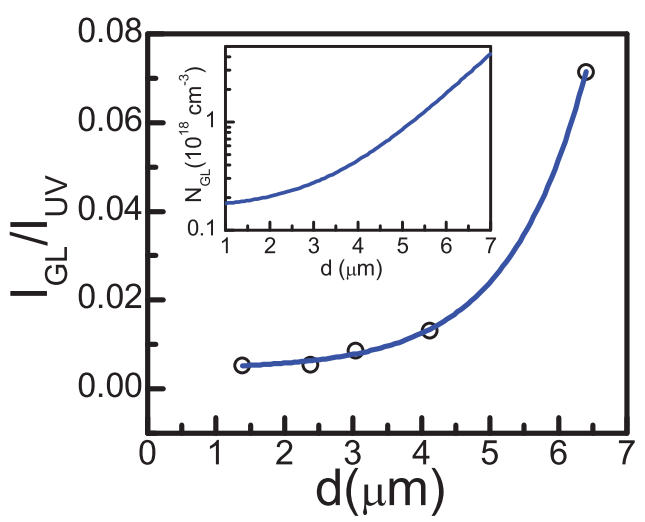

FIG. 3. Ratio of GL intensity to the intensity of the UV emission as a function of the diameter of the microwire. The line represents the fit using Eq. (6). The inset shows the $\mathrm{N}_{G L}$ profile obtained from this fitting.

the microwire, since the GL comes from a surface layer of $\approx 30 \mathrm{~nm}$ [Ref. 8] and the UV penetration is about $100 \mathrm{~nm}$ [Ref. 18]. The quantum efficiencies can be evaluated by the following expressions: ${ }^{18}$

$$
\begin{gathered}
\eta_{U V}=f_{r} \frac{k_{U V}}{k_{U V}+\left(\frac{N_{G L}}{N_{G L}+N_{U V}}\right) k_{G L}+\left(\frac{N_{N R}}{N_{N R}+N_{U V}}\right) k_{N R}}, \\
\eta_{G L}=\frac{\left(\frac{N_{G L}}{N_{G L}+N_{U V}}\right) k_{G L}}{k_{U V}+\left(\frac{N_{G L}}{N_{G L}+N_{U V}}\right) k_{G L}+\left(\frac{N_{N R}}{N_{N R}+N_{U V}}\right) k_{N R}},
\end{gathered}
$$

where $\mathrm{N}_{U V} \mathrm{~N}_{N R}$, and $\mathrm{N}_{G L}$ are the number density of photoexcited excitons (UV), non-radiative defects (NR), and green emitting defects (GL), respectively. $\mathrm{k}_{U V} \mathrm{k}_{N R}$, and $\mathrm{k}_{G L}$ are the rate constants associated with radiative recombination of excitons at the band edge (UV), nonradiative recombination of excitons (NR), and transfer of energy from excitons to green-emitting defects (GL), respectively. The factor $\mathrm{f}_{r}$ in Eq. (4) accounts for the fraction of the UV photons emitted by excitons that were not reabsorbed, while green emission experiences no such parasitic reabsorption. Replacing Eqs. (4) and (5) in (3),

$$
\frac{I_{G L}}{I_{U V}}=K f_{r} \frac{k_{G L}}{k_{U V}} \frac{N_{G L}}{N_{G L}+N_{U V}},
$$

where $\mathrm{K}$ include the constants of Eq. (3). The $\mathrm{N}_{U V}$ is constant since it depends on the energy and fluence of the laser and on the reflectivity and optical absorbance of the $\mathrm{ZnO} .^{18}$ Then the variation of the $\mathrm{I}_{G L} / \mathrm{I}_{U V}$ ratio comes mainly from the variation of the $\mathrm{N}_{G L}$ along the microwire. Using Eq. (6), we fitted the data of $\mathrm{I}_{G L} / \mathrm{I}_{U V}$ vs. d (see Fig. 3) by proposing a profile of defect concentration as: $\mathrm{N}_{G L}(d)=\mathrm{A}+\mathrm{B}^{*} \exp (\mathrm{d} / \mathrm{C})$, where $\mathrm{A}, \mathrm{B}$, and $\mathrm{C}$ are the fitting parameters. It is possible to obtain an approximate quantitative profile for $\mathrm{N}_{G L}$ assuming the values for $\mathrm{f}_{r}=0.11,1 / \mathrm{k}_{U V}=2 \mathrm{~ns}$, and $1 / \mathrm{k}_{G L}=2.53 \mathrm{~ns}$ obtained by Simmons et al. ${ }^{18}$ Since our $\mu \mathrm{PL}$ setup has a continuous wave photoexcitation is very complex the determination of the $\mathrm{N}_{U V}$ due to carrier-exciton equilibration effects. Nevertheless, a rough approximation of $\mathrm{N}_{U V}$ can be estimated if we note in Fig. 2 that the intensity of the UV emission grows when the spot goes from pos. 1 to pos. 2, i.e., when the $\mathrm{N}_{G L}$ decreases from the maximum value. This means that in pos. 1 , it is reaching the condition where $N_{G L} \approx 0.1 N_{U V}^{18}$ and $\eta_{U V}$ drops while $\eta_{G L}$ grows. As we shall see below, from the resistance vs. temperature measurements, we estimate a defect concentration of $4 \times 10^{18} \mathrm{~cm}^{-3}$. Considering the latter estimation, we approximate $N_{U V} \approx 10^{20} \mathrm{~cm}^{-3}$. In the inset of Fig. 3, it is shown the fitted $\mathrm{N}_{G L}$ profile. The density of deep defects changes one order of magnitude from the base to the tip of the microwire.

On the other hand, the UV peak of Fig. 2 is sharper than GL band. It is attributed to the exciton recombination or the band edge transition that could involve shallow donor levels. In this case, we observed a red shift of the UV peak as the diameter of the wires increase (see Fig. 4). This behaviour suggests the narrowing of the gap energy. It is reasonable to think that as well as the density of deep levels increase for larger diameter, the density of other type of defects will also increase with a similar exponential profile. We can fit the experimental data of Fig. 4 using a dependence on the diameter following an exponential function. From this fit, we found $\mathrm{E}_{g}=3.33(1) \mathrm{eV}$ by extrapolation to zero diameter. This value is close to the gap value for bulk $\mathrm{ZnO}{ }^{4}$ We consider that there is a combined effect of lattice distortion ${ }^{20,21}$ and an increased tailing of the absorption edge $\mathrm{e}^{22}$ in the microwire, which are causing the red shift of the band gap edge. An asymmetric lattice distortion can establish a tensile stress field along the microwire that can also be established by a gradient in the vacancies concentration. While the change in the tailing of the absorption edge is due to the variation of the concentration of shallow defects along the microwire.

Fig. 5 shows the PR spectra at room temperature of a microwire contacted using nanolithography (sample 2, see Figure 1(b)). The PR is defined as $\mathrm{PR}(\%)=\left(\mathrm{R}_{i}-\mathrm{R}_{d}\right) / \mathrm{R}_{d} \times$ 100 , where $R_{i}$ is the resistance under illumination and $R_{d}$ is the resistance in dark. The wavelength sweep rate used was $1 \mathrm{~nm} / \mathrm{s}$. Before initiating the experiments, we reach the saturation value of the resistance using $\lambda=250 \mathrm{~nm}(4.96 \mathrm{eV})$ for $2 \mathrm{~h}$. The wavelength sweeps were repeated several times under the same initial conditions in order to check the repeatability and were confirmed by obtaining the same result. We distinguish in these spectra two minima: One at $2.65 \mathrm{eV}$ $(469 \mathrm{~nm})$ and other at $2.32 \mathrm{eV}(535 \mathrm{~nm})$ due to promoted photocarriers to deep states or CB. The minimum

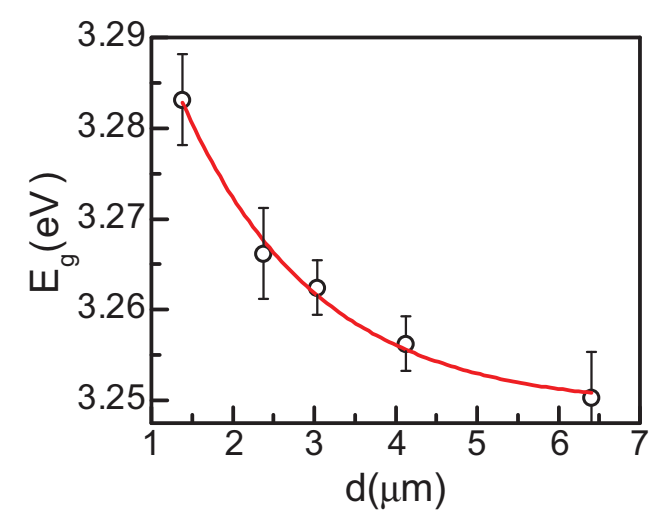

FIG. 4. UV peak energy vs. the diameter of the microwire. The line represents the fit of the data using an exponential function (see text). 


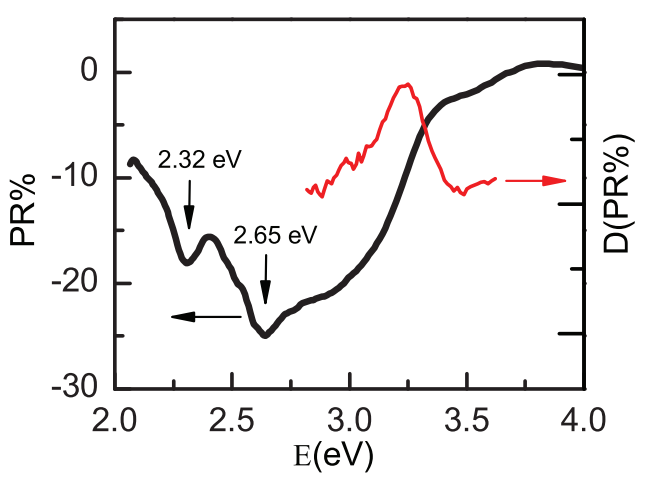

FIG. 5. Photoresistance of the sample 2 as a function of the energy of the incident light at room temperature. Inset, derivative of the data (right vertical axis) around the near band gap energy.

corresponding to the band gap is overwhelmed by the minimum of $2.65 \mathrm{eV}$, nevertheless, the derivative of the spectra has a maximum at around $3.25 \mathrm{eV}$, see inset in Fig. 5 (right vertical axis). The same experiment was done for sample 1, contacted by cold soldering. The spectra obtained in this sample are noisy, and it is difficult to distinguish any structure in them. Nevertheless, there is a clear minimum that is systematically repeated and defined at $3.25 \mathrm{eV}$ that coincides with the value for the UV peak obtained by $\mu \mathrm{PL}$ on the thicker zone of the MW. This minimum of the PR spectrum has its origin in the promoted photocarriers from the $\mathrm{VB} /$ deep levels to the $\mathrm{CB} /$ levels near the bottom of $\mathrm{CB}$.

It is worth to point out that the information that give an excitation spectrum (PR) is complementary to an emission spectrum (PL). Comparing the results from $\mu \mathrm{PL}$ and PR excitation spectra, we found a coincidence at $2.32 \mathrm{eV}$ within an error of $0.05 \mathrm{eV}$. While the minimum observed at $2.65 \mathrm{eV}$ in $\mathrm{PR}$ is not present in the $\mu \mathrm{PL}$ spectra. The origin of this minimum is discussed below.

Fig. 6 shows the variation of the normalized resistance, $\mathrm{R} / \mathrm{R}_{300 K}$, with the temperature of sample 2 in the dark. $\mathrm{R}_{300 K}$ is the resistance at $300 \mathrm{~K}$. Inspired by the model of Mott and Davis, ${ }^{23}$ we propose for the resistivity

$$
\rho(T)^{-1}=\rho_{0}^{-1}+\rho_{A}^{-1} e^{-\epsilon_{A} / K_{B} T}+\rho_{B}^{-1} e^{-\epsilon_{B} / K_{B} T},
$$

where the activation energy $\epsilon_{A}$ is the energy required to promote an electron into the conduction band and $\epsilon_{B}$ is the

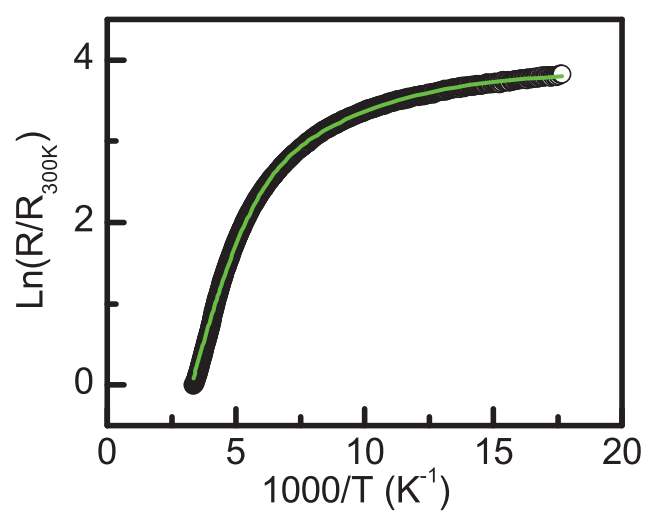

FIG. 6. Normalized dark resistance of the sample 2 as a function of the temperature. The line represents the fit of the data using Eq. (7).

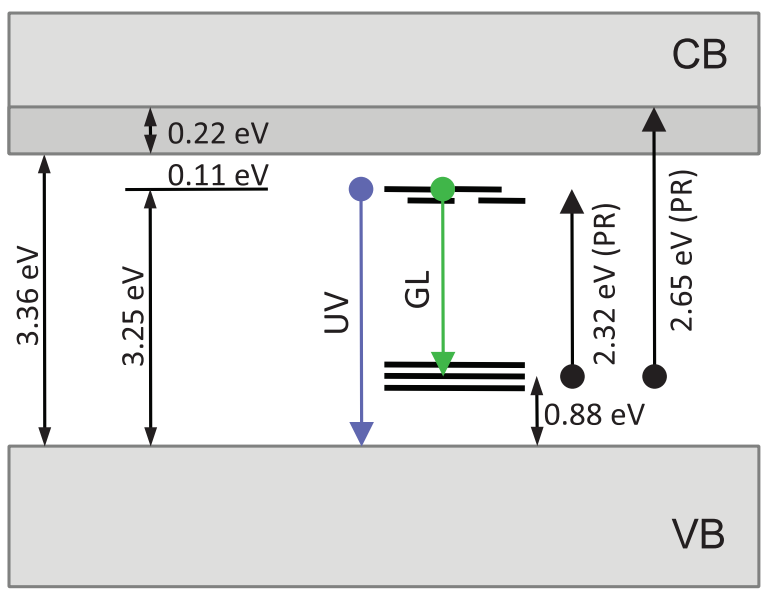

FIG. 7. Schematic diagram of the energy levels in $\mathrm{ZnO}$ obtained in this work.

activation energy for nearest neighbor hopping conduction in the impurity band. The temperature-independent term $\rho_{0}^{-1}$ represents the residual resistance measurable at low enough temperatures. Fitting of the experimental data gives an activation energy $\epsilon_{A}=110 \mathrm{meV}$ and $\epsilon_{B}=26 \mathrm{meV}$. The defect density can be evaluated using $\epsilon_{B}$ and it gives $4 \times 10^{18} \mathrm{~cm}^{-3}{ }^{24}$ This value is rather high and it makes sense since the variation of the resistance in the whole range of temperature measured is only around fifty times.

In Fig. 7, we propose an energy band schematics that describes the combined information given by the experiments. The RT measurement in sample 2 shows that there are levels at $110 \mathrm{meV}$ below the CB. Surface defects could be the source of this band that contributes to the tailing of the $\mathrm{CB}$. These levels are tentatively attributed to $\mathrm{Zn}_{i}^{+2}$ in the literature. ${ }^{14,25}$ From the UV emission of PL, we infer that the $\mathrm{VB}$ is at $3.25 \mathrm{eV}$ below these levels (UV arrow in Fig. 7). Also, there are deep levels located at $2.37 \mathrm{eV}$ below these centers, corresponding to $0.88 \mathrm{eV}$ from the VB (GL arrow in Fig. 7). These levels correspond to $V_{Z n}^{-2}$ defects. $^{4,26}$ Indeed, the doubly negatively charged state of the zinc vacancy has the lowest formation energy when the Fermi level is near the bottom of the $\mathrm{CB} .{ }^{11}$ Moreover, it has been demonstrated that the zinc vacancy is the dominant acceptor defect in as-grown n-type $\mathrm{ZnO} .^{27}$ From the PR excitation measurements, we can deduce that the electrons are promoted from the deep levels up to about $0.22 \mathrm{eV}$ on the top of the filling states in the $\mathrm{CB}$ (2.65 eV-PR arrow in Fig. 7). On the other hand, PR also shows that can promote electrons from deep states to the centers near the bottom of the CB (2.32 eV-PR arrow in Fig. 7), then by thermalization these electrons go to the upper levels where they participate in the electronic transport.

\section{CONCLUSIONS}

In summary, we experimentally studied and evaluated the point defects in single $\mathrm{ZnO}$ microwires grown by carbothermal reduction. We were able to find the deep level density profile and the gap narrowing as a function of the defect concentration along the microwires. The photoresistance spectra show a characteristic deep defect level inside the gap at $0.88 \mathrm{eV}$ from top of the VB corresponding to 
$V_{Z n}^{-2}$. The resistance as a function of the temperature show defect levels next to the bottom of the CB $(110 \mathrm{meV})$ and a defect concentration of $4 \times 10^{18} \mathrm{~cm}^{-3}$. This combination of techniques allows us to study the density of defects profile, gap size, and states of defects inside the gap in single $\mathrm{ZnO}$ microwires.

\section{ACKNOWLEDGMENTS}

This work was supported by CIUNT under Grant No. 26/E439 and by FONARSEC FSTICS $002 / 2010$ and by the collaborative project SFB 762 "Functionality of Oxide Interfaces." We thank C. Czekalla for the provision of some of the samples used in this work. F.I. acknowledges the CAPES, CNPq, and FAPESP for financial support.

${ }^{1}$ D. C. Look, J. W. Hemsky, and J. Sizelove, Phys. Rev. Lett 82, 2552 (1999). ${ }^{2}$ X. L. Xu, S. P. Lau, J. S. Chen, G. Y. Chen, and B. K. Tay, J. Cryst. Growth 223, 201 (2001).

${ }^{3}$ J. Carrasco, N. Lopez, and F. Illas, Phys. Rev. Lett. 93, 225502 (2004).

${ }^{4}$ H. Morkoc and U. Ozgur, Zinc Oxide, Fundamentals, Materials, and Device Technology, 1st ed. (Wiley-VCH Verlag GmbH \& Co. KGaA, Federal Republic of Germany, 2009).

${ }^{5}$ C. G. V. der Walle, Phys. Rev. Lett. 85, 1012 (2000).

${ }^{6}$ K. M. Wong, S. M. Alay-e-Abbas, Y. Fang, A. Shaukat, and Y. Lei, J. Appl. Phys. 114, 034901 (2013).

${ }^{7}$ S. S. Brenner, J. Appl. Phys. 27, 1484 (1956).

${ }^{8}$ I. Shalish, H. Temkin, and V. Narayanamurti, Phys. Rev. B 69, 245401 (2004).
${ }^{9}$ F. Leiter, H. Alves, A. Hofstaetter, D. Hofmann, and B. Meyer, Phys. Status Solidi B 226, R4 (2001).

${ }^{10}$ K. Vanheusden, W. L. Warren, C. H. Seager, D. R. Tallant, J. A. Voigt, and B. E. Gnade, J. Appl. Phys. 79, 7983 (1996).

${ }^{11}$ A. F. Kohan, G. Ceder, D. Morgan, and C. G. Van de Walle, Phys. Rev. B 61, 15019 (2000).

${ }^{12}$ A. Janotti and C. G. Van de Walle, Phys. Rev. B 76, 165202 (2007).

${ }^{13}$ B. Lin, Z. Fu, and Y. Jia, Appl. Phys. Lett. 79, 943 (2001).

${ }^{14}$ M. Liu, A. Kitai, and P. Mascher, J. Lumin. 54, 35 (1992).

${ }^{15}$ R. Vidya, P. Ravindran, H. Fjellvåg, B. G. Svensson, E. Monakhov, M. Ganchenkova, and R. M. Nieminen, Phys. Rev. B 83, 045206 (2011).

${ }^{16}$ R. Dingle, Phys. Rev. Lett. 23, 579 (1969).

${ }^{17}$ Y. Alivov, M. Chukichev, and V. Nikitenko, J. Semicond. 38, 31 (2004).

${ }^{18}$ J. G. Simmons, J. V. Foreman, J. Liu, and H. O. Everitt, Appl. Phys. Lett. 103, 201110 (2013)

${ }^{19}$ B. Q. Cao, M. Lorenz, M. Brandt, H. von Wenckstern, J. Lenzner, G. Biehne, and M. Grundmann, Phys. Status Solidi RRL 2, 37 (2008).

${ }^{20}$ W. Qin, T. Nagase, Y. Umakoshi, and J. A. Szpunar, J. Phys.: Condens. Matter 19, 236217 (2007).

${ }^{21}$ C. P. Dietrich, M. Lange, F. J. Klpfel, H. von Wenckstern, R. SchmidtGrund, and M. Grundmann, Appl. Phys. Lett. 98, 031105 (2011).

${ }^{22}$ J. Wang, Z. Wang, B. Huang, Y. Ma, Y. Liu, X. Qin, X. Zhang, and Y. Dai, ACS Appl. Mater. Interfaces 4, 4024 (2012).

${ }^{23}$ N. Mott and E. Davis, Electronic Processes in Non-Crystalline Materials, 2nd ed. (University Press, Oxford, 1979).

${ }^{24}$ B. Shklovskii and A. Efros, Electronic Properties of Doped Semiconductors, Solid-State Science Vol. 45 (Springer-Verlag, 1984).

${ }^{25}$ L. Schmidt-Mende and J. MacManus-Driscoll, Mater. Today 10, 40 (2007).

${ }^{26}$ T. M. Börseth, B. G. Svensson, A. Y. Kuznetsov, P. Klason, Q. X. Zhao, and M. Willander, Appl. Phys. Lett. 89, 262112 (2006).

${ }^{27}$ F. Tuomisto, V. Ranki, and K. Saarinen, Phys. Rev. Lett. 91, 205502 (2003). 